
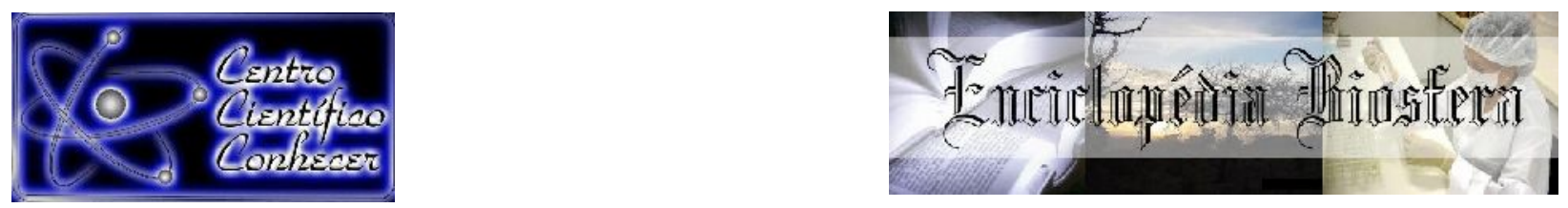

\title{
PREVALÊNCIA DA SÍNDROME DE BURNOUT EM AGENTES COMUNITÁRIOS DE SAÚDE DA ATENÇÃO PRIMÁRIA NO MUNICIPIO DE CACHOEIRO DE ITAPEMIRIM-ES
}

Marcelo Costa Vicente ${ }^{1}$; Arlindo José Freire Portes ${ }^{2}$

1 Enfermeiro. Mestre em Saúde da Família. Professor Faculdades Integradas São

Pedro. E-mail: enfmarcelovicente@gmail.com

2 Médico. Doutorado em Oftalmologia. Professor da Universidade Estácio de Sá.

Recebido em: 22/09/2018 - Aprovado em: 23/11/2018 - Publicado em: 03/12/2018 DOI: 10.18677/EnciBio_2018B125

A síndrome de Burnout tem como característica principal a resposta referente à exaustão profissional resultante do contato direto e permanente de relações intensas das atividades laborais e no atendimento a pessoas, sendo classificada em três tipos ou fatores: exaustão emocional; a despersonalização e a decepção. $O$ objetivo dessa pesquisa foi avaliar a dimensão da Síndrome de Burnout em agentes comunitários de saúde que trabalham na atenção primária no município de Cachoeiro de Itapemirim-ES. Tratou-se de uma pesquisa transversal envolvendo 144 profissionais agentes comunitários de saúde. Para caracterizar a prevalência e a representação social dos agentes, foi empregado um questionário sociocultural e a Escala padronizada de Caracterização de Burnout para analisar a magnitude da síndrome. Para a análise estatística foi utilizado o programa Epiinfo7 com análise descritiva, bivariada e multivariada. A prevalência da Síndrome foi de $34,72 \%$ (IC95\%: 26,94 - 42,5); a estatística relativa dos fatores de risco aos níveis altos e baixos foi de $15,98 \%$ e para os coeficientes médios e baixos, foi de $49,30 \%$. Das variáveis da pesquisa associadas à Síndrome, a principal foi: escolaridade (OR: 8,18 IC95\% 1,04-64,31) e a falta ao trabalho (OR: 0,45. IC95\%: 0,22 -0,94). Existe uma prevalência significativa para a síndrome de Burnout em agentes comunitários de saúde, diante desses resultados, existe uma necessidade de medidas preventivas e de intervenção urgentes, como forma de aperfeiçoar a qualidade do ambiente ocupacional.

PALAVRAS-CHAVE: Agentes Comunitários de Saúde. Esgotamento profissional. Prevalência. Saúde do trabalhador.

\section{PREVALENCE OF BURNOUT SYNDROME IN COMMUNITY HEALTH AGENTS OF PRIMARY ATTENTION IN CACHOEIRO CITY OF ITAPEMIRIM-ES}

\begin{abstract}
Burnout syndrome has as its main characteristic the response related to professional exhaustion resulting from direct and permanent contact of intense work relationships in the care of people, being classified into three types or factors: emotional exhaustion; depersonalization and deception. The objective of this research was to evaluate the dimension of Burnout Syndrome in community health agents that
\end{abstract}


perform primary care in the city of Cachoeiro de Itapemirim-ES. It was a crosssectional study involving 144 professional community health agents. To characterize the prevalence and social representation of the agents, a socio-demographic questionnaire and the standardized Burnout Characterization Scale were used to analyze the syndrome event. For the statistical analysis, the Epiinfo7 program was performed with descriptive, bivariate and multivariate analysis. The prevalence of the syndrome was $34.72 \%(95 \% \mathrm{Cl}: 26.94-42.5)$; the relative statistic of the risk factors at high and low levels was $15.98 \%$ and for the mean and low coefficients it was 49.30\%. The main variables were: schooling (OR: 8.18 95\% Cl $1.04-64.31$ ) and emotional exhaustion was: lack of work (OR: $0.45,95 \% \mathrm{Cl}: 0.22-0.94)$. There is a significant prevalence for Burnout syndrome in community health agents. Given these results, there is a need for urgent preventive and intervention measures as a way to improve the quality of the occupational environment.

KEYWORDS: Health Community Workers. Burnout Professional. Prevalence Occupational Health.

\section{INTRODUÇÃO}

As modificações dos sistemas de saúde advindas da globalização, introduzidas nas relações de trabalho, permitiram às instituições de saúde em seus mais diversos setores, o aumento e reorganização das estruturas produtivas. Entretanto, esse novo processo produtivo acompanhado do desenvolvimento técnico científico trouxe impactos significativos à saúde dos trabalhadores, acarretando aparecimento de doenças no âmbito físico e psíquico, entre as quais se menciona o estresse (CARLOTTO et al., 2013).

O termo estresse refere à patologia intrinsecamente pautada ao modo de reação do profissional frente ao agente causador, é representado por episódios da vida e fontes internas, tais como a forma de pensar, valores e crenças. Portanto, o estresse vem acometendo de forma mais incisiva o profissional por reflexos advindos diretamente do ambiente no qual ele convive e as diversas condições de trabalho envolvidas (KUSHNIR et al., 2014).

Aprofundando o entendimento sobre o estresse, o profissional de saúde agente comunitário de saúde estando constantemente em contato com fatores estressantes, existe a possibilidade de desenvolver a Síndrome de Burnout, a qual pode atingir inúmeras profissões, em qualquer faixa etária, se destacando principalmente entre profissionais que tem maiores contatos interpessoais (FREIRE; TRENTIN, 2016).

A Síndrome de Burnout é um problema psíquico, reconhecido como um dos grandes problemas psicossociais que inutiliza a qualidade de vida de profissionais de qualquer área, estando mais predisposto aquelas que envolvem cuidados com saúde, ocasionando um importante transtorno ocupacional e desordem social aos trabalhadores (CARLOTTO et al, 2013). Essa síndrome envolve três dimensões: exaustão emocional, despersonalização e baixa realização profissional ou falta de envolvimento pessoal no trabalho, ou ainda, decepção (SILVA et al., 2017a).

As dimensões são caracterizadas como: exaustão emocional refere ao sentimento de fadiga, aumento do desânimo e fraqueza, um esgotamento das energias, sendo diminuída a ponto de tornar-se incapaz de executar suas tarefas do cotidiano na prática laboral (BEZERRA; ARAGÃO, 2014). A desumanização é o fator interpessoal, que engloba maneiras negativas, indiferenças ou impaciência, chegando a ocorrer o afastamento do profissional com os seus pacientes (SILVA; 
MENEZES, 2008). Por último tem a realização profissional diminuída, por sua vez, é um sentimento de inaptidão, incapacidade, incapacidade da execução de seu trabalho, o profissional pode ter a percepção do papel do seu trabalho como ineficaz, acreditando que seus serviços não têm valor, aumentando o absenteísmo (LIMA et al., 2018).

Diversos estudos científicos associaram a Síndrome de Burnout com o trabalho de profissionais de saúde em geral e principalmente os profissionais de saúde que atuam na Estratégia Saúde da Família (ESF), entre eles, encontram-se as profissões da equipe mínima com funções assistenciais, como, por exemplo, os profissionais da enfermagem, médicos, dentistas, auxiliar de consultório dentário e agentes comunitários de saúde (ACS) (MARTINS et al., 2014).

A categoria, que tem maior número de profissionais por unidade e com diversas tarefas distintas, especificamente é o agente comunitário de saúde, onde a Síndrome de Burnout está diretamente associada à relação desse profissional com a população de sua área que, por muitas vezes, apresentam permeados de dúvidas e conflitos, carga horária elevada, jornadas trabalhistas duplas, idade mais elevada, estado civil, dentre outros. Toda essa realidade pode desvirtuar o trabalhador para sentimentos de ansiedade/agonia e, em momentos mais crônicos, a ausência da capacidade de apresentar soluções viáveis aos problemas vividos no dia a dia (RODRIGUES et al., 2018).

De acordo com Mota et al.,(2014) durante toda a execução das atividades no trabalho o agente comunitário dentro da Estratégia de Saúde da Família, está diretamente ligado a diversos fatores que contribuem para o esgotamento profissional, sendo; número diminuído de profissionais por área, somado às demandas elevadas de trabalho; excesso e variedade de atividades executadas; salários inferiores aos demais profissionais da atenção primária, acarretando duplas ou triplas jornadas de trabalho; carga horária semanal desgastante; inexistência de materiais de trabalho mínimo para execução das tarefas; aumento de cobranças e diminuição do reconhecimento do trabalho pela sociedade. Além das dificuldades extras do local de trabalho, como problemas de ordem familiar e social, que interferem negativamente na qualidade de vida no trabalho.

O aparecimento da Síndrome de Burnout entre os profissionais da atenção primária em saúde está cada vez mais presente em publicações científicas atuais, porém este tipo de trabalho científico é escasso quando são relacionados aos agentes comunitários de saúde. No dia a dia, os agentes comunitários de saúde, se deparam com ambientes mais perigosos, insalubres e favoráveis a riscos à saúde. Eles ficam diretamente expostos a fatores sociais e ambientais na micro área de atuação, sem dispor de alguns recursos básicos para prestação de uma assistência digna à comunidade; somada as falhas presentes na rede de atenção à saúde que irá refletir diretamente no exercício profissional do agente, propiciando o desenvolvimento da síndrome de Burnout e de outras doenças psíquicas relacionadas ao trabalho (MOTA et al., 2014). Neste contexto de discussão, a presente pesquisa teve como objetivo avaliar a ocorrência da Síndrome de Burnout em Agentes Comunitários de Saúde que atuam na Estratégia Saúde da Família no município de Cachoeiro de Itapemirim-ES.

\section{MATERIAL E MÉTODOS}

Tratou-se de estudo descritivo de natureza transversal, de base populacional. Foram colhidos dados primários socais por meio de questionário, com 12 perguntas 
fechadas, semiestruturado com dados sócios demográficos e Escala da Categorização do Burnout, no período de junho a julho de 2014. O projeto de pesquisa foi aprovado pelo Comitê de Ética e Pesquisa (CAAE: 31009414.7.0000.5284). Foram entrevistados 144 dos 176 agentes comunitários de saúde atuantes nas 43 equipes de Saúde da Família, nos bairros e distritos do município de Cachoeiro de Itapemirim. Não participaram dessa pesquisa 32 agentes comunitários de saúde, que no momento da pesquisa estavam de férias, licença ou não quiseram participar.

A coleta de dados foi realizada no próprio local de trabalho, com agendamentos prévios com data e horário com o enfermeiro da unidade. A atenção primária em Saúde de Cachoeiro de Itapemirim-ES atua na modalidade de Gestão Plena da Atenção Básica, tendo um Teto da Cobertura da Atenção Primária que reúne $71 \%$ de cobertura com a Estratégia de Agentes Comunitários de Saúde e 54\% de Estratégia Saúde da Família (SESA, 2014). Adotou-se como instrumento de coleta de dados, dois questionários: No primeiro questionário foram coletados dados pessoais e características profissionais e do trabalho, com o objetivo de descrever as variáveis do estudo. Já o segundo questionário utilizou a Escala de Caracterização do Burnout (ECB). O questionário da Escala de Categorização de Burnout apresenta as seguintes dimensões: a Exaustão Emocional, Desumanização e a Realização Pessoal Diminuída.

Para classificação das dimensões, os pontos de corte foram calculados a partir dos percentis da distribuição, adotando como referência o padrão de resposta da própria amostragem, tal como sugerido por Maslach e Jackson (1996). Considerando estas recomendações, os indivíduos acima do percentil 75 foram enquadrados em grau alto de presença de Burnout; entre os percentis 25 e 75, grau moderado; e abaixo de 25, grau leve.

A análise estatística dos dados foi realizada através do Software Epiinfo 7.2 (CDC Atlanta, EUA). O software R foi empregado complementarmente. A estatística descritiva das variáveis incluiu: frequências, médias, percentis ou desvios padrões. Foi realizada regressão logística binomial simples e múltipla com as variáveis: escolaridade, população de risco, uso ou não de medicamentos, sexo, falta ao trabalho, faixa etária e tipo de religião. O estudo utilizou a técnica de passo para trás ("backstep"), em que uma variável por vez foi eliminada até que todas as variáveis restantes possuíssem $p<0,05$. A variável eliminada a cada passo era a que apresentava maior valor de $\mathrm{p}$.

\section{RESULTADOS}

A prevalência da Síndrome de Burnout apresentada neste estudo foi de $34,72 \%$ (de 144) entre os ACS atuantes em Cachoeiro de Itapemirim no ES. No total dos 144 Agentes Comunitários de Saúde do município de Cachoeiro de ItapemirimES, foram observados que a maioria (77) possuia idade acima de 42 anos $(53,4 \%)$, seguida pela faixa etária de 30 a 35 anos $(27,77 \%)$. Ao analisar a variável sexo, 139 $(96,52 \%)$ são trabalhadores do sexo feminino e cinco $(3,47 \%)$ do sexo masculino.

Em relação a variável estado civil, $97(67,36 \%)$ dos entrevistados são casados, 24 (16,66\%) são solteiros, 12 (8,33\%) são divorciados, nove (6,25\%) vivem com o companheiro e duas $(1,38)$ são viúvas. Em relação a variável escolaridade, entre os profissionais $122(84,72 \%)$ tem o ensino médio completo, apenas 15 $(10,41 \%)$ tem o ensino fundamental completo e sete $(4,86 \%)$ tem o ensino superior completo. Na distribuição quantitativa da religião a pesquisa revelou que quase 
todos são cristãos, ou seja, $95(65,97 \%)$ têm crença voltada ao catolicismo, 42 $(29,16 \%)$ são protestantes e quatro $(2,77 \%)$ tem outras crenças e três $(2,08 \%)$ declararam que não tem religião. Sobre a distribuição das variáveis em relação ao salário, a fala foi unânime: Não estão satisfeitos com o salário. Quanto ao resultado da pesquisa em relação ao tempo de exercício da profissão como agente comunitário de saúde, observou-se que 142 (98,61\%) têm mais de cinco anos de tempo de trabalho e dois $(1,39 \%)$ tem dois anos de exercício da profissão, estando esses profissionais mais propício para o acometimento da Síndrome de Burnout.

Ao perguntar aos agentes comunitários de saúde se já participaram de alguma capacitação no local de trabalho, a pesquisa revelou que $140(97,22 \%)$ já participaram e quatro (2,77\%) não participaram de nenhuma capacitação. Em relação ao absenteísmo, à pesquisa revelou que $80(55,55 \%)$ nunca faltaram às atividades do trabalho e $64(44,44 \%)$ já se ausentaram do serviço por atestado e licenças. Em relação as famílias cadastradas em sua área de atuação, em situação de risco, a pesquisa revelou que $99(68,75 \%)$ dos agentes comunitários de saúde trabalham em áreas de risco, como áreas violentas, riscos de desmoronamentos e alagamentos e $45(31,25 \%)$ não trabalham nestes locais. De acordo com os entrevistados, quanto ao uso de medicação controlada, a pesquisa revelou que 86 $(59,72 \%)$ não fazem uso de medicação sob controle e 58 (40,27\%) fazem uso de remédios com indicação médica como os tranquilizantes e antidepressivos.

Do total de agentes comunitários pesquisados, 34,72\% apresentaram níveis moderados e altos para as três dimensões da síndrome, $15,98 \%$ possuíam um nível alto, um nível médio e um nível baixo e $49,30 \%$ tinham apenas níveis médios e baixos para as três dimensões pesquisadas. Foram ponderados como portadores da Síndrome, os agentes que apresentavam exclusivamente níveis altos e moderados das dimensões estudadas.

TABELA 1: Análise do resultado final da regressão logística múltipla com os fatores de risco estudados.

\begin{tabular}{lccccccc}
\multicolumn{1}{c}{ Termo } & $\begin{array}{c}\text { Razão de } \\
\text { Chance }\end{array}$ & $\begin{array}{c}\text { IC95\% } \\
\text { Min }\end{array}$ & Max & Coeficiente & $\begin{array}{c}\text { Erro } \\
\text { Padrão. }\end{array}$ & $\begin{array}{c}\text { Estatística } \\
\text { Z }\end{array}$ & p-valor \\
\hline $\begin{array}{l}\text { Escolaridade } \\
(2 / 1)\end{array}$ & 8,1818 & 1,0409 & 64,3113 & 2,1019 & 1,0520 & 1,9981 & 0,0457 \\
\hline $\begin{array}{l}\text { Escolaridade } \\
\text { 3/1) }\end{array}$ & 0,0000 & $0,0000>1.0 \mathrm{E} 12$ & $-11,7502$ & 305,4217 & $-0,0385$ & 0,9693 \\
\hline CONSTANTE & ${ }^{*}$ & $*$ & $*$ & $-2,6391$ & 1,0351 & $-2,5496$ & 0,0108 \\
\hline
\end{tabular}

O resultado da regressão logística binomial múltipla revelou que a única variável que apresentava significância estatística $(p=0,045)$ para fator de risco da Síndrome de Burnout foi escolaridade, ou seja, o fato do profissional passar a ter escolaridade equivalente ao nível médio em relação ao nível fundamental, como é preconização mínima do Ministério da Saúde (Escolaridade 2/1) aumenta a chance de apresentar a Síndrome de Burnout em oito vezes (RC: 8,18; IC95\% 1,04 - 64,31). $\mathrm{O}$ aumento da razão de chance relacionado à comparação do nível de escolaridade superior com o nível fundamental foi prejudicado pelo baixo número de agentes de saúde neste nível de escolaridade, portanto, esta categoria de variável escolaridade não foi considerada para o resultado válido (Escolaridade 3/1).

TABELA 2: Análise de regressão logística simples de cada característica sócio ENCICLOPÉDIA BIOSFERA, Centro Científico Conhecer - Goiânia, v.15 n.28; p.1323 
cultural e profissional dos agentes comunitários de saúde em relação aos fatores da Síndrome de Burnout, no município de Cachoeiro de Itapemirim-ES.

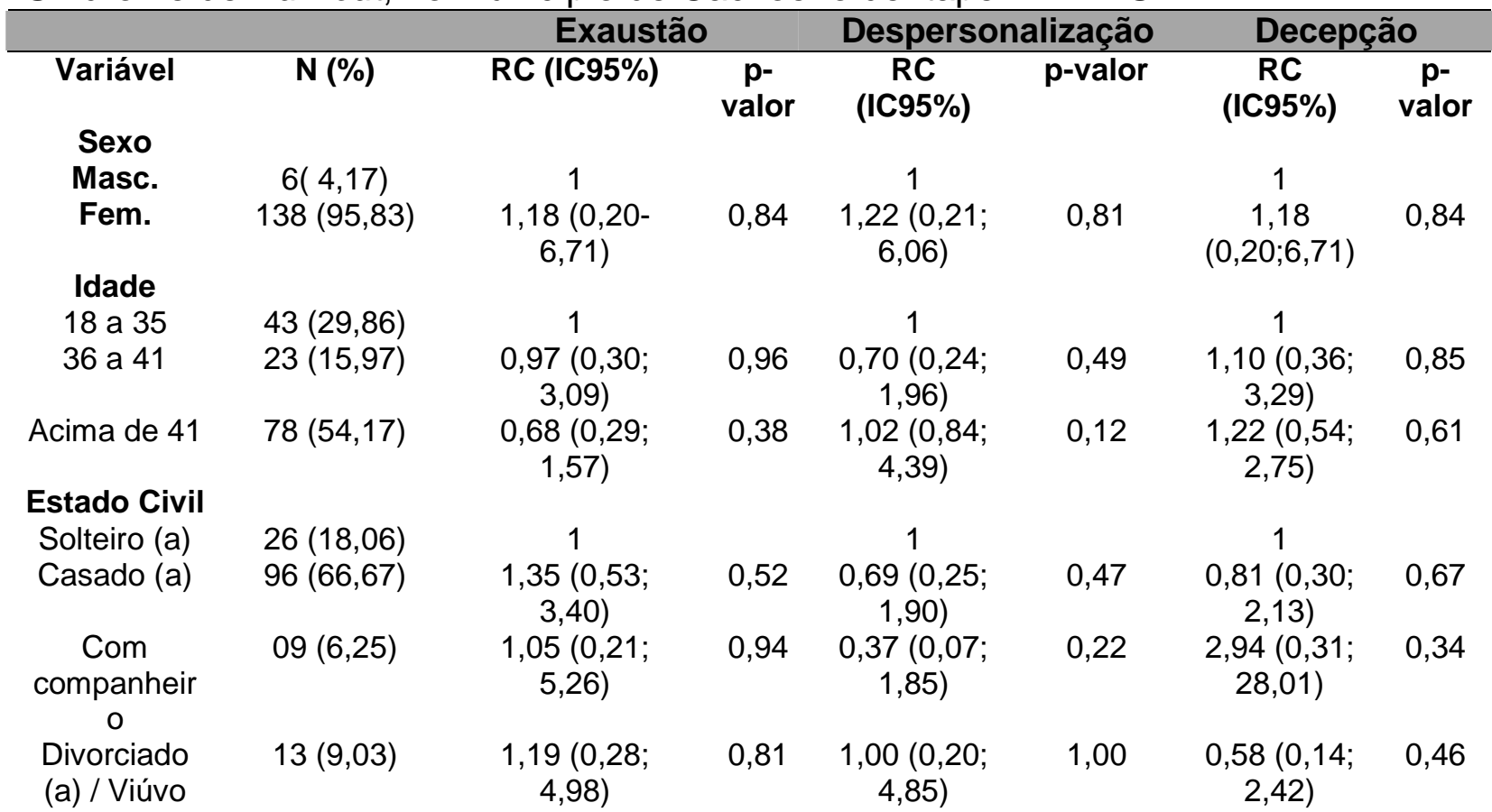

(a)

\begin{tabular}{|c|c|c|c|c|c|c|c|}
\hline \multicolumn{8}{|l|}{ Religião } \\
\hline Católico & $92(63,99)$ & 1 & & 1 & & & \\
\hline Protest. & $44(30,56)$ & $\begin{array}{c}0,62(0,06 ; \\
6,25)\end{array}$ & 0,68 & $\begin{array}{c}0,68(0,06 ; \\
6,90)\end{array}$ & 0,75 & $\begin{array}{c}2,83(0,37 \\
21,24)\end{array}$ & 0,75 \\
\hline Sem religião & $4(2,78)$ & $\begin{array}{c}1,29(0,12 \\
13,98)\end{array}$ & 0,83 & $\begin{array}{c}1,29(0,12 \\
13,98)\end{array}$ & 0,83 & $\begin{array}{c}1,93(0,24 \\
15,11)\end{array}$ & 0,83 \\
\hline Outros & $4(2,78)$ & $\begin{array}{c}1,00(0,04 ; 24 \\
54)\end{array}$ & 1,00 & $\begin{array}{c}0,33(0,01 ; \\
6,65)\end{array}$ & 0,47 & $\begin{array}{c}1,00(0,06 \\
15,98)\end{array}$ & 0,47 \\
\hline \multicolumn{8}{|l|}{$\begin{array}{c}\text { Escolaridad } \\
e\end{array}$} \\
\hline Ensino fund. & $15(10,42)$ & 1 & & 1 & & 1 & \\
\hline $\begin{array}{l}\text { Ensino } \\
\text { médio }\end{array}$ & $122(84,72)$ & $\begin{array}{c}1,95(0,64 \\
5,94)\end{array}$ & 0,23 & $\begin{array}{c}0,38(0,08 \\
1,78)\end{array}$ & 0,22 & $\begin{array}{c}3,21(1,07 ; \\
9.57)\end{array}$ & 0,03 \\
\hline $\begin{array}{l}\text { Ensino } \\
\text { superior }\end{array}$ & $7(4,86)$ & $\begin{array}{c}0,11(0,01 \\
1,17)\end{array}$ & 0,06 & $\begin{array}{c}0,06(0,00 \\
0,56)\end{array}$ & 0,01 & $\begin{array}{c}1,52(0,24 \\
9,29)\end{array}$ & 0,64 \\
\hline \multicolumn{8}{|l|}{$\begin{array}{l}\text { Faltou ao } \\
\text { trabalho }\end{array}$} \\
\hline Não & & 1 & & 1 & & 1 & \\
\hline Sim & $64(44,44)$ & $\begin{array}{c}0,45(0,22 ; \\
0,94)\end{array}$ & 0,03 & $\begin{array}{c}1,25(0,60 \\
2,60)\end{array}$ & 0,53 & $\begin{array}{c}1,52(0,70 \\
3,10)\end{array}$ & 0,25 \\
\hline \multicolumn{8}{|l|}{$\begin{array}{l}\text { População } \\
\text { de risco }\end{array}$} \\
\hline Não & & 1 & & 1 & & 1 & \\
\hline Sim & $98(68,06)$ & $\begin{array}{c}1,04(0,48 \\
1,23)\end{array}$ & 0,09 & $\begin{array}{c}1,27(0,53 ; \\
2,72)\end{array}$ & 0,53 & $\begin{array}{c}0,55(0,24 \\
1,24)\end{array}$ & 0,15 \\
\hline
\end{tabular}

Observa-se na tabela acima que, apenas o fator absenteísmo gerou significância estatística para a exaustão emocional, (RC: 0,45; IC95\% - 0,22 - 0,94; $\mathrm{p}=0,03$ ). Outro ponto a ser considerado e que ocasionou significância estatística para despersonalização, foi escolaridade ao realizar comparação do nível ensino superior em relação ao ensino fundamental (RC: 0,06; IC95\% - 0,00-0,56; $p=0,01$ ). 
Em relação à dimensão decepção, apenas a variável que apresentou significância estatística, foi Escolaridade referente ao ensino médio em relação ao ensino fundamental (RC: 3,$21 ;$ IC95\%: 1,07-9,57; $p=0,03$ ).

\section{DISCUSSÃO}

$\mathrm{Na}$ presente pesquisa foi encontrado índice considerável de agentes comunitários de saúde com a Síndrome de Burnout. Apesar do valor quantitativo da amostra estudada ter sido suficiente, consideram ainda que existam elementos que dificultam a resposta adequada às perguntas do questionário, podendo omitir dados, obtendo atitudes defensivas dos profissionais sobre o contexto de trabalho (CHICONATTO et al., 2018).

Em relação aos dados, da faixa etária, o estudo revelou uma população acima de 42 anos. Os resultados assemelham-se aos resultados obtidos na pesquisa de Leite et al., (2014) em que os profissionais com maior idade têm por características ser profissionais com certa maturidade profissional e maior administração em situações estressantes.

Ao analisar a variável sexo, observou-se a predominância de profissionais do sexo feminino. Esse número elevado de pessoas do sexo feminino no processo de trabalho é constatado e evidenciado no cotidiano das unidades assistências de saúde (SILVA et al., 2017a).

Em relação ao estado civil, a maior parte dos participantes são casados, para o desencadeamento para a Síndrome de Burnout, a vida conjugal é um fator de risco, pelo fato que as mulheres casadas têm além do trabalho diário, atividades domésticas e os cuidados com cônjuge e filhos (CHICONATTO et al., 2018).

Observou-se no estudo quanto à escolaridade, que grande parte tem o ensino médio completo. Estudos de Jodas e Haddad (2009) revelou que quanto maior é a escolaridade, maiores são as chances de apresentar altos escores para a síndrome de Burnout e outro fator importante que pode ser pertinente é a ausência do reconhecimento e status em determinadas profissões. Para os agentes comunitários de saúde, o nível de escolaridade deveria ser o mesmo grau de instrução da comunidade onde o profissional atua em suas atividades laborativas, uma vez que a população ficaria com o mesmo nível sociocultural do profissional que o assiste.

$\mathrm{Na}$ distribuição da religião a pesquisa evidenciou que a maioria tem sua crença voltada ao catolicismo. A religiosidade é uma disposição de cada ser humano como forma de amenizar os problemas negativos do dia a dia, buscando coragem e conforto direto às respostas a fim de minimizar o problema vivenciado no local de trabalho (SANTOS, 2008).

Em relação ao tipo de vínculo empregatício, observou-se que todos eram contrato temporário, ou seja, sem carteira assinada, precedido de processos seletivos, existindo contratos que se renovam anualmente. $\mathrm{O}$ fato dos profissionais não terem um vínculo durável, ou seja, estável, não existe uma garantia trabalhista, podendo afetar o rendimento das atividades laborais (BORGES et al.,2005).

Quanto ao resultado da pesquisa, em relação ao tempo de exercício da profissão, observou-se que grande parte dos entrevistados têm mais de cinco anos de trabalho. É uma das características que induz os profissionais a estarem dentro do perfil dos fatores de risco, pois o fato do elevado tempo de trabalho, associado ao tempo de contato intenso com a mesma população, problemas burocráticos das atividades profissionais, pacientes e seus familiares pode induzir à síndrome (CHICONATTO et al., 2018). 
Observou-se que a maioria dos agentes comunitários de saúde já participaram de determinado curso de capacitação no local de trabalho. Quando os profissionais participam de capacitações, qualificação e/ou aperfeiçoamento, ele coopera para o seu desenvolvimento e a realização profissional (GODOI et al., 2018).

Notou-se que a maioria dos agentes comunitários de saúde tem em sua micro área, famílias cadastradas em área de situação risco, como desmoronamentos, enchentes, tráfico dentre outras. Esses profissionais apresentam elevados fatores predisponentes para a síndrome do esgotamento profissional (BARROSO; GUERRA, 2013).

\section{CONCLUSÕES}

A presente pesquisa confirma a prevalência de Síndrome de Burnout em agentes comunitários de saúde, pois esses profissionais trabalham em áreas estressantes e condições precárias, causando grande desgaste emocional. A Estratégia Saúde da Família vem se consolidando junto ao Sistema único de Saúde, aprimorando o acolhimento e assistência aos pacientes que buscam atendimento à saúde. Diante dessas complexidades, as demandas e cargas de trabalho dos agentes comunitários são fatores que favorecem o aparecimento de problemas psíquicos, como a Síndrome de Burnout.

Promover e manter a qualidade de vida no trabalho é um desafio constante para os agentes comunitários que assumem a função de assistência direta a comunidade. Analisando a importância do trabalho do ACS na saúde pública, há a necessidade de realização de novos estudos com esta categoria, principalmente em outras regiões do estado e do Brasil com o objetivo de abranger mais aspectos do processo de trabalho e da possível prevalência da Síndrome de Burnout em agentes comunitários.

\section{REFERÊNCIAS}

BARROSO, S.M.; GUERRA, A.R.P. Burnout e qualidade de vida de agentes comunitários de saúde de Caetanópolis (MG). Caderno de Saúde Coletiva, Rio de Janeiro, v. 21, n. 3, p. 338-345. 2013.

BEZERRA, M.J.; ARAGÃO, A.E.A. Síndrome de Burnout e o trabalho de enfermeiros emergencistas de um hospital de ensino da zona norte do estado do ceará. Sobral v. 1, n. 4, p.: 60-74. 2014. Disponível em: <http://www.inta.com.br/ biblioteca/images/pdf/art-5-rev-4.pdf>.

BORGES, L.O.; TAMAYO, A.; ALVES FILHO, A. Significado do trabalho entre trabalhadores de saúde. In: Borges LO. Os trabalhadores de saúde e seu trabalho. São Paulo: Casa do Psicólogo, 2005.

CARLOTTO, S.; PIZZINATO, A.; ROCHA, K.B.; MACHADO, R.O. Prevalence and factors associated with burnout syndrome in professionals in basic health units. Ciencia \& Trabajo, Léon, v. 15, n. 47, p. 76-80. 2013. Disponível em: <http://www.scielo.cl/pdf/cyt/v15n47/art07.pdf>. Acesso em: 06 set. 2018.

CHICONATTO. P.; SCHMITT, V.; BERNARDI, L.; MENON, M.U.; NOVELLO, D. Percepção sobre a existência de trabalho interdisciplinar em estratégias saúde da 
família da cidade-sede da $5^{\text {a }}$ Regional de Saúde do Paraná. Revista da Universidade Vale do Rio Verde, v. 16, n. 1, p. 1, jan./jul. 2018.

FREIRE, P.L.; TRENTIN, J.P. Trends in burnout syndrome and emotional factors: an assessment of anesthesiologists in Southern Brazil, 2012. Psychol Health Med. v 21, n. 4, p. 413-23. 2016. DOI: http://dx.doi.org/10.1080/ 13548506.2016.1139143

GODOI, B.B; MADEIRA, A.L.F; ALFRADIQUE, B.M; DOMINGOS, G.P.; BRUGIOLO, I.F.; et al. Capacitação de agentes comunitários de saúde no município de Diamantina - MG. Revista Ciência em Extensão. v.14, n.1, p.54-69. 2018.

JODAS, D.A.; HADDAD, M.C.L. Síndrome de Burnout em trabalhadores de enfermagem de um pronto socorro de hospital universitário. Acta Paulista de Enfermagem, v. 22, n.2, p.192-7. 2009.

KUSHNIR, T.; GREENBERG, D.; MADJAR, N.; HADARI, I.; YERMIAHU, Y.; et al. Is burnout associated with referral rates among primary care physicians in community clinics? Family Practice, v. 31, n. 1, p. 44-50. 2014.

LEITE, D.F.; NASCIMENTO, D.D.G.; OLIVEIRA, M.A.C. Qualidade de vida no trabalho de profissionais do NASF no município de São Paulo. Physis Revista de Saúde Coletiva, RJ, v 24, n. 2, p. 507-525. 2014.

LIMA, A.S.; FARAH, B.F.; TEIXEIRA, M.T.B. Análise da prevalência da Síndrome de Burnout em profissionais da atenção primária em saúde. Trabalho, Educação e Saúde, v.16, n. 1, Rio de Janeiro Jan./Apr. 2018.

MASLACH, C.; JACKSON, M.P. The Maslach Burnout Inventory: Test manual. Palo Alto, CA. Consulting Psycologist Press, 3 ed., 1996.

MARTINS, L.F.; LAPORT, T.J.; MENEZES, V.P.; MEDEIROS, P.B.; RONZANI, T.M. Esgotamento entre profissionais da Atenção primária à Saúde. Ciência da Saúde Coletiva, v. 19, n. 12, p. 4739-50. 2014. DOI: http://dx.doi.org/10.1590/1413812320141912.03202013

MOTA, C. M.; DOSEA, G. S.; NUNES, P. S. Avaliação da presença da Síndrome de Burnout em Agentes Comunitários de Saúde no município de Aracaju, Sergipe, Brasil. Ciência \& Saúde Coletiva, Rio de Janeiro, v. 19, n. 12, p. 4719-4726. 2014.

RODRIGUES, L.S; SANTANA, S.M; OLIVEIRA, G.F. A Síndrome de Burnout no Contexto da ESF: Uma Análise das suas Dimensões. Id on Line Revista Multidisciplinar e Psicologia, v.12, n. 39. 2018.

SANTOS, G.; A.C. As interpretações do mal na religião e a Síndrome de Burnout. Revista da Abordagem gestáltica, v.14, n. 1, p.57-65. 2008

SESA-ES. Secretaria Estadual de Saúde do Espírito Santo. [homepage na internet]. Plano Diretor de Regionalização; 2014 Disponível em: 
http://www.saude.es.gov.br/download/FichaMunicipal2013_Cl.pdf.

SILVA, A.T.C.; MENEZES, P.R. Esgotamento profissional e transtornos mentais comuns em agentes comunitários de saúde. Revista Saúde Pública, v. 42, n. 5, p. 921-929. 2008.

SILVA, J.P.C.; FERREIRA, L.S.; ALMEIDA, B.L.F. A nova organização do trabalho e a saúde do trabalhador $5^{\circ}$ Encontro Internacional de Política Social e $12^{\circ}$ Encontro Nacional de Política Social. Vitória (ES, Brasil), 5 a 8 de junho de 2017. Disponível em < http://www.periodicos.ufes.br/EINPS/article/view/16572.

SILVA, D.K.C.; PACHECO, M.J.T.; MARQUES, H.S.; BRANCO, R.C.C.; SILVA, M.A.C.N.; et al. Burnout no trabalho de médicos pediatras. Revista Brasileira Medicina do Trabalho. v 15, n 1, p.2-11, 2017. 